\title{
New Čebyšev Type Inequalities and Applications for Functions of Self-Adjoint Operators on Complex Hilbert Spaces
}

\author{
Mohammad W. Alomari \\ Department of Mathematics, Faculty of Science and Information Technology, Jadara University, Irbid 21110, Jordan \\ Correspondence should be addressed to Mohammad W. Alomari; mwomath@gmail.com
}

Received 28 January 2014; Accepted 20 May 2014; Published 5 June 2014

Academic Editor: Prasanna Kumar Sahoo

Copyright (c) 2014 Mohammad W. Alomari. This is an open access article distributed under the Creative Commons Attribution License, which permits unrestricted use, distribution, and reproduction in any medium, provided the original work is properly cited.

Several new error bounds for the Čebyšev functional under various assumptions are proved. Applications for functions of selfadjoint operators on complex Hilbert spaces are provided as well.

\section{Introduction}

In recent years the approximation problem of the RiemannStieltjes integral $\int_{a}^{b} f(t) d u(t)$ via the famous Čebyšev functional

$$
\begin{aligned}
\mathscr{T}(f, g)= & \frac{1}{b-a} \int_{a}^{b} f(t) g(t) d t \\
& -\frac{1}{b-a} \int_{a}^{b} f(t) d t \cdot \frac{1}{b-a} \int_{a}^{b} g(t) d t
\end{aligned}
$$

increasingly became essential. In 1882, Čebyšev [1] derived an interesting result involving two absolutely continuous functions whose first derivatives are continuous and bounded and is given by

$$
|\mathscr{T}(f, g)| \leq \frac{1}{12}(b-a)^{2}\left\|f^{\prime}\right\|_{\infty}\left\|g^{\prime}\right\|_{\infty}
$$

and the constant $1 / 12$ is the best possible.

In 1935, Grüss [2] proved another result for two integrable mappings $f, g$ such that $\phi \leq f(x) \leq \Phi$ and $\gamma \leq f(x) \leq \Gamma$; the inequality

$$
|\mathscr{T}(f, g)| \leq \frac{1}{4}(\Phi-\phi)(\Gamma-\gamma)
$$

holds, and the constant $1 / 4$ is the best possible.
In [3, p 302] Beesack et al. have proved the following Čebyšev inequality for absolutely continuous functions whose first derivatives belong to $L_{p}$ spaces:

$$
\begin{aligned}
& |\mathscr{T}(f, g)| \\
& \quad \leq \frac{b-a}{4}\left(\frac{2^{p}-1}{p(p+1)}\right)^{1 / p}\left(\frac{2^{q}-1}{q(q+1)}\right)^{1 / q}\left\|f^{\prime}\right\|_{p}\left\|g^{\prime}\right\|_{q},
\end{aligned}
$$

where $\|h\|_{p}:=\left(\int_{a}^{b}|h(t)|^{p} d t\right)^{1 / p}, \forall p>1$, and $(1 / p)+(1 / q)=1$.

For the constant

$$
\omega(p, q):=\frac{1}{4}\left(\frac{2^{p}-1}{p(p+1)}\right)^{1 / p}\left(\frac{2^{q}-1}{q(q+1)}\right)^{1 / q}
$$

we have

$$
\frac{1}{8} \leq \omega(p, q) \leq \frac{1}{4}
$$

for all $q=p /(p-1), p>1$. Furthermore, we have the following particular cases in (4).

(1) If $p=q=2$, we have

$$
|\mathscr{T}(f, g)| \leq \frac{b-a}{8}\left\|f^{\prime}\right\|_{2}\left\|g^{\prime}\right\|_{2} .
$$

(2) If $q \rightarrow \infty$, we have

$$
|\mathscr{T}(f, g)| \leq \frac{b-a}{4}\left\|f^{\prime}\right\|_{1}\left\|g^{\prime}\right\|_{\infty} .
$$


In 1970, Ostrowski [4] has proved the following combination of the Čebyšev and Grüss results:

$$
|\mathscr{T}(f, g)| \leq \frac{1}{8}(b-a)(M-m)\left\|g^{\prime}\right\|_{\infty}
$$

where $g$ is absolutely continuous with $g^{\prime} \in L_{\infty}[a, b]$ and $f$ is Lebesgue integrable on $[a, b]$ and satisfying $m \leq f(t) \leq M$, for all $t \in[a, b]$. The constant $1 / 8$ is the best possible.

In 1973, Lupaş [5] has improved Beesack et al. inequality (7), as follows:

$$
|\mathscr{T}(f, g)| \leq \frac{(b-a)}{\pi^{2}}\left\|f^{\prime}\right\|_{2}\left\|g^{\prime}\right\|_{2}
$$

provided that $f, g$ are two absolutely continuous functions on $[a, b]$ with $f^{\prime}, g^{\prime} \in L_{2}[a, b]$, where $\left\|h^{\prime}\right\|_{2}:=\left(\int_{a}^{b}\left|h^{\prime}(t)\right|^{2} d t\right)^{1 / 2}$. The constant $1 / \pi^{2}$ is the best possible.

More recently, and using the identity ([3], page 246),

$$
\begin{aligned}
\mathscr{T}(f, g)=\frac{1}{b-a} \int_{a}^{b} & {\left[f(t)-\frac{f(a)+f(b)}{2}\right] } \\
& \times\left[g(t)-\frac{1}{b-a} \int_{a}^{b} g(s) d s\right] d t .
\end{aligned}
$$

Dragomir [6] has proved the following inequality.

Theorem 1. Let $f:[a, b] \rightarrow \mathbb{C}$ be of bounded variation on $[a, b]$ and $g:[a, b] \rightarrow \mathbb{C}$ a Lebesgue integrable function on $[a, b]$; then

$$
\begin{aligned}
& |\mathcal{T}(f, g)| \\
& \quad \leq \frac{1}{2} \bigvee_{a}^{b}(f) \cdot \frac{1}{b-a} \int_{a}^{b}\left|g(t)-\frac{1}{b-a} \int_{a}^{b} g(s) d s\right| d t
\end{aligned}
$$

where $\bigvee_{a}^{b}(f)$ denotes the total variation of $f$ on the interval $[a, b]$. The constant $1 / 2$ is best possible in (12).

Another result when both functions are of bounded variation was considered in the same paper [6], as follows.

Theorem 2. If $f, g:[a, b] \rightarrow \mathbb{C}$ are of bounded variation on $[a, b]$, then

$$
|\mathscr{T}(f, g)| \leq \frac{1}{4} \bigvee_{a}^{b}(f) \cdot \bigvee_{a}^{b}(g) .
$$

The constant $1 / 4$ is best possible in (13).

Many authors have studied the functional (1) and, therefore, several bounds under various assumptions have been obtained; for more new results and generalizations the reader may refer to [6-21].

On other hand and in order to study the difference between two Riemann integral means, Barnett et al. [22] have proved the following estimates.
Theorem 3. Let $f:[a, b] \rightarrow \mathbb{R}$ be an absolutely continuous function with the property that $f^{\prime} \in L_{\infty}[a, b]$; that is,

$$
\left\|f^{\prime}\right\|_{\infty}:=\operatorname{ess} \sup _{t \in[a, b]}\left|f^{\prime}(t)\right| .
$$

Then for $a \leq c<d \leq b$, we have the inequality

$$
\begin{aligned}
\mid \frac{1}{b-a} & \int_{a}^{b} f(t) d t-\frac{1}{d-c} \int_{c}^{d} f(s) d s \mid \\
\leq & {\left[\frac{1}{4}+\left(\frac{(a+b) / 2-(c+d) / 2}{(b-a)-(d-c)}\right)^{2}\right] } \\
& \times[(b-a)-(d-c)]\left\|f^{\prime}\right\|_{\infty} \\
\leq & \frac{1}{2}[(b-a)-(d-c)]\left\|f^{\prime}\right\|_{\infty} .
\end{aligned}
$$

The constant $1 / 4$ in the first inequality and $1 / 2$ in the second inequality are the best possible.

After that, Cerone and Dragomir [23] have obtained the following three results as well.

Theorem 4. Let $f:[a, b] \rightarrow \mathbb{R}$ be an absolutely continuous mapping. Then for $a \leq c<d \leq b$, we have the inequalities

$$
\begin{aligned}
& \left|\frac{1}{b-a} \int_{a}^{b} f(t) d t-\frac{1}{d-c} \int_{c}^{d} f(s) d s\right| \\
& \leq \begin{cases}\frac{(b-a)}{(q+1)^{1 / q}}\left[1+\left(\frac{\rho}{1+\rho}\right)^{q}\right]^{1 / q} & f^{\prime} \in L_{p}[a, b], \\
\times\left[v^{q+1}+\lambda^{q+1}\right]^{1 / q}\left\|f^{\prime}\right\|_{p}, & 1 \leq p<\infty, \\
& \frac{1}{p}+\frac{1}{q}=1 ; \\
\frac{1}{2}[1-\rho+|v-\lambda|]\left\|f^{\prime}\right\|_{1}, & f^{\prime} \in L_{1}[a, b],\end{cases}
\end{aligned}
$$

where $(b-a) v=c-a,(b-a) \rho=d-c$ and $(b-a) \lambda=b-d$. Both inequalities in (16) are sharp.

Theorem 5. Assume that the mapping $f:[a, b] \rightarrow \mathbb{R}$ is of $r$-H-Hölder type on $[a, b]$. Then for $a \leq c<d \leq b$, we have the inequality

$$
\begin{gathered}
\left|\frac{1}{b-a} \int_{a}^{b} f(t) d t-\frac{1}{d-c} \int_{c}^{d} f(s) d s\right| \\
\leq H \frac{(c-a)^{r+1}+(b-d)^{r+1}}{(r+1)[(b-a)-(d-c)]}
\end{gathered}
$$

Inequality (17) is best possible in the sense that we cannot put in the right-hand side a constant less than 1. 
Theorem 6. Let $f:[a, b] \rightarrow \mathbb{R}$ be a mapping of bounded variation on $[a, b]$. The following bounds hold:

$$
\begin{aligned}
& \left|\frac{1}{b-a} \int_{a}^{b} f(t) d t-\frac{1}{d-c} \int_{c}^{d} f(s) d s\right| \\
& \leq\left\{\begin{array}{c}
{\left[\frac{b-a-(d-c)}{2}+\left|\frac{c+d}{2}-\frac{a+b}{2}\right|\right] \frac{\vee_{a}^{b}(f)}{b-a}} \\
L \frac{(c-a)^{2}+(b-d)^{2}}{2[(b-a)-(d-c)]} \\
\text { if } f \text { is L-Lipschitzian } \\
\left(\frac{b-d}{b-a}\right) f(b)-\left(\frac{c-a}{b-a}\right) f(a) \\
+\left[\frac{c+d-(a+b)}{b-a}\right] f\left(s_{0}\right) ; \\
\text { if } f \text { is monotonic nondecreasing, }
\end{array}\right.
\end{aligned}
$$

where $s_{0}=(c b-a d) /((b-a)-(d-c)) \in[c, d]$.

In this paper by utilising amongst others the inequalities from Theorems 3-6, several new bounds for the Čebyšev functional $\mathscr{T}(f, g)$ are provided.

The inequalities (15)-(18) are used in an essential way to obtain new error bounds for the $\mathscr{T}(f, g)$, which gives a significant application for these inequalities. Applications for functions of self-adjoint operators on complex Hilbert Spaces are provided as well.

\section{The Case When $f$ Is of Bounded Variation}

We may start with the following result.

Theorem 7. Let $f, g:[a, b] \rightarrow \mathbb{R}$ be such that $f$ is of bounded variation on $[a, b]$ and $g$ is absolutely continuous on $[a, b]$; then

$$
\begin{aligned}
& |\mathcal{T}(f, g)| \\
& \quad \leq \bigvee_{a}^{b}(f) \cdot \begin{cases}\frac{1}{8}(b-a)\left\|g^{\prime}\right\|_{\infty}, & g^{\prime} \in L_{\infty}[a, b] ; \\
\frac{1}{2}\left(\frac{1+\left(1 / 2^{q}\right)}{2(q+1)}\right)^{1 / q}\left\|g^{\prime}\right\|_{p^{\prime}}, & g^{\prime} \in L_{p}[a, b] ; \\
\frac{1}{8}\left\|g^{\prime}\right\|_{1}, & g^{\prime} \in L_{1}[a, b],\end{cases}
\end{aligned}
$$

where $\|\cdot\|_{p}$ are the usual Lebesgue norms; that is,

$$
\begin{aligned}
\|h\|_{p}:= & \left(\int_{a}^{b}|h(t)|^{p} d t\right)^{1 / p}, \quad \text { for } p \geq 1, \\
& \|h\|_{\infty}:=\text { ess } \sup _{t \in[a, b]}|h(t)| .
\end{aligned}
$$

Proof. Using integration by parts, we have

$$
\mathscr{T}(f, g)
$$

$$
=-\frac{1}{b-a} \int_{a}^{b}\left(\int_{a}^{t} g(s) d s-\frac{t-a}{b-a} \int_{a}^{b} g(s) d s\right) d f(t) .
$$

It is known that for a continuous function $p:[a, b] \rightarrow \mathbb{R}$ and a function $v:[a, b] \rightarrow \mathbb{R}$ of bounded variation, the Riemann-Stieltjes integral $\int_{a}^{b} p(t) d \nu(t)$ exists and one has the inequality

$$
\left|\int_{a}^{b} p(t) d \nu(t)\right| \leq \sup _{t \in[a, b]}|p(t)| \bigvee_{a}^{b}(\nu)
$$

As $f$ is of bounded variation on $[a, b]$, by $(22)$ we have

$$
\begin{array}{r}
\left|-\frac{1}{b-a} \int_{a}^{b}\left(\int_{a}^{t} g(s) d s-\frac{t-a}{b-a} \int_{a}^{b} g(s) d s\right) d f(t)\right| \\
\leq \frac{1}{b-a} \sup _{\mid}\left|\int_{a}^{t} g(s) d s-\frac{t-a}{b-a} \int_{a}^{b} g(s) d s\right| \cdot \bigvee_{a}^{b}(f) \\
=\frac{1}{b-a} \sup _{t \in[a, b]} \mid(t-a)\left[\frac{1}{t-a} \int_{a}^{t} g(s) d s\right. \\
\left.-\frac{1}{b-a} \int_{a}^{b} g(s) d s\right] \mid \bigvee_{a}^{b}(f) .
\end{array}
$$

In the inequality (15), setting $d=t$ and $c=a$, we get

$$
\left|\frac{1}{t-a} \int_{a}^{t} g(s) d s-\frac{1}{b-a} \int_{a}^{b} g(s) d s\right| \leq \frac{1}{2}(b-t)\left\|g^{\prime}\right\|_{\infty} .
$$

Substituting (24) into (23), we get

$$
\begin{aligned}
|\mathcal{T}(f, g)| & \leq \frac{1}{b-a} \cdot \frac{1}{2}\left\|g^{\prime}\right\|_{\infty} \sup _{t \in[a, b]}\{(t-a)(b-t)\} \bigvee_{a}^{b}(f) \\
& =\frac{1}{8}(b-a)\left\|g^{\prime}\right\|_{\infty} \bigvee_{a}^{b}(f),
\end{aligned}
$$

since $\sup _{t \in[a, b]}\{(t-a)(b-t)\}$, occurs at $t=(a+b) / 2$, therefore, $\sup _{t \in[a, b]}\{(t-a)(b-t)\}=(1 / 4)(b-a)^{2}$, which proves the first inequality in (19).

In the inequality (16), setting $d=t$ and $c=a$, we get

$$
\begin{aligned}
& \left|\frac{1}{t-a} \int_{a}^{t} g(s) d s-\frac{1}{b-a} \int_{a}^{b} g(s) d s\right| \\
& \quad \leq \begin{cases}\frac{(b-t)^{1+(1 / q)}}{(q+1)^{1 / q}(b-a)^{1 / q}} \\
\times\left[1+\left(\frac{t-a}{b+t-2 a}\right)^{q}\right]^{1 / q}\left\|g^{\prime}\right\|_{p^{\prime}} & g^{\prime} \in L_{p}[a, b], \\
\frac{1}{2} \frac{b-t}{b-a}\left\|g^{\prime}\right\|_{1}, & g^{\prime} \in L_{1}[a, b] .\end{cases}
\end{aligned}
$$


Substituting (26) into (23), we get

$|\mathscr{T}(f, g)|$

$$
\begin{aligned}
& \leq \frac{1}{b-a} \bigvee_{a}^{b}(f) \\
& \times \sup _{t \in[a, b]}(t-a) \\
& \begin{cases}\frac{(b-t)^{1+(1 / q)}}{(q+1)^{1 / q}(b-a)^{1 / q}} & \\
\times\left[1+\left(\frac{t-a}{b+t-2 a}\right)^{q}\right]^{1 / q}\left\|g^{\prime}\right\|_{p}, & g^{\prime} \in L_{p}[a, b], \\
\frac{1}{2} \frac{b-t}{b-a}\left\|g^{\prime}\right\|_{1}, & g^{\prime} \in L_{1}[a, b]\end{cases} \\
& \leq \bigvee_{a}^{b}(f) \times \begin{cases}\frac{\left[1+(1 / 2)^{q}\right]^{1 / q}}{2^{1+1 / q}(1+q)^{1 / q}}\left\|g^{\prime}\right\|_{p^{\prime}}, & g^{\prime} \in L_{p}[a, b] ; \\
\frac{1}{8}\left\|g^{\prime}\right\|_{1}, & g^{\prime} \in L_{1}[a, b],\end{cases}
\end{aligned}
$$

where $p>1$ and $(1 / p)+(1 / q)=1$, which proves the second and the third inequalities in (19).

Another result when $g$ is of $r$-H-Hölder type is as follows.

Theorem 8. Let $f, g:[a, b] \rightarrow \mathbb{R}$ be such that $f$ is of bounded variation on $[a, b]$ and $g$ is of $r-H$-Hölder type on $[a, b]$; then

$$
|\mathscr{T}(f, g)| \leq \frac{H r^{r}}{(r+1)^{r+1}}(b-a)^{r} \bigvee_{a}^{b}(f) .
$$

Proof. As $f$ is of bounded variation and $g$ is of $r$-H-Hölder type on $[a, b]$, by $(23)$ and using (17) we have

$$
\begin{aligned}
& |\mathscr{T}(f, g)| \\
& \leq \frac{1}{b-a} \sup _{t \in[a, b]} \mid(t-a)\left[\frac{1}{t-a} \int_{a}^{t} g(u) d u\right. \\
& \left.-\frac{1}{b-a} \int_{a}^{b} g(u) d u\right] \mid \bigvee_{a}^{b}(f) \\
& \leq \frac{1}{b-a} \frac{H}{r+1} \sup _{t \in[a, b]}(t-a)(b-t)^{r} \bigvee_{a}^{b}(f) \\
& =H \frac{r^{r}}{(r+1)^{r+1}}(b-a)^{r} \bigvee_{a}^{b}(f),
\end{aligned}
$$

since $\sup _{t \in[a, b]}(t-a)(b-t)^{r}=\left(r^{r} /(r+1)^{r+1}\right)(b-a)^{r+1}$, which completes the proof.

Theorem 9. Let $f, g:[a, b] \rightarrow \mathbb{R}$ be such that $f$ is of bounded variation on $[a, b]$ and $g$ is monotonic nondecreasing on $[a, b]$; then

$$
|\mathscr{T}(f, g)| \leq \frac{1}{6}(b-a)[g(b)-g(a)] \bigvee_{a}^{b}(f) .
$$

Proof. As $f$ and $g$ is of bounded variation on $[a, b]$ and $g$ is monotonic nondecreasing on $[a, b]$, by (22) and using (23) we have

$$
\begin{aligned}
&|\mathscr{T}(f, g)| \leq \frac{1}{b-a} \int_{a}^{b} \mid(t-a) \\
& \qquad \frac{1}{t-a} \int_{a}^{t} g(u) d u \\
&\left.\quad-\frac{1}{b-a} \int_{a}^{b} g(u) d u\right] \mid d t \bigvee_{a}^{b}(f) .
\end{aligned}
$$

In the third part of inequality (18), setting $d=t$ and $c=a$, we get

$\left|\frac{1}{t-a} \int_{a}^{t} g(s) d s-\frac{1}{b-a} \int_{a}^{b} g(s) d s\right| \leq \frac{b-t}{b-a}[g(b)-g(a)]$.

Substituting (32) into (31), we get

$$
\begin{aligned}
|\mathscr{T}(f, g)| & \leq \frac{1}{b-a} \cdot \frac{g(b)-g(a)}{b-a} \int_{a}^{b}(t-a)(b-t) d t \bigvee_{a}^{b}(f) \\
& =\frac{1}{6}(b-a)[g(b)-g(a)] \bigvee_{a}^{b}(f),
\end{aligned}
$$

and thus the proof is finished.

\section{The Case When $f$ Is Lipschitzian}

In this section, we give some new bounds when $f$ is $L$ Lipschitzian.

Theorem 10. Let $f, g:[a, b] \rightarrow \mathbb{R}$ be such that $f$ is $L$ Lipschitzian on $[a, b]$ and $g$ is an absolutely continuous on $[a, b]$; then

$$
\begin{aligned}
& |\mathscr{T}(f, g)| \\
& \quad \leq L \begin{cases}\frac{1}{12}(b-a)^{2}\left\|g^{\prime}\right\|_{\infty}, & g^{\prime} \in L_{\infty}[a, b] ; \\
\frac{q^{2}\left(1+\left(1 / 2^{q}\right)\right)^{1 / q}}{(2 q+1)(3 q+1)(q+1)^{1 / q}} & \\
\times(b-a)^{2}\left\|g^{\prime}\right\|_{p} & g^{\prime} \in L_{p}[a, b] ; \\
\frac{1}{12}(b-a)\left\|g^{\prime}\right\|_{1} & g^{\prime} \in L_{1}[a, b],\end{cases}
\end{aligned}
$$

where $p>1$ and $(1 / p)+(1 / q)=1$.

Proof. Using the fact that for a Riemann integrable function $p:[c, d] \rightarrow \mathbb{R}$ and L-Lipschitzian function $v:[c, d] \rightarrow \mathbb{R}$, one has the inequality

$$
\left|\int_{c}^{d} p(t) d \nu(t)\right| \leq L \int_{c}^{d}|p(t)| d t .
$$


As $f^{\prime}$ is $L$-Lipschitzian on $[a, b]$, by (35) we have

$$
\begin{aligned}
|\mathscr{T}(f, g)| \leq \frac{L}{b-a} \int_{a}^{b} \mid(t-a)\left[\frac{1}{t-a} \int_{a}^{t} g(u) d u\right. \\
\left.\quad-\frac{1}{b-a} \int_{a}^{b} g(u) d u\right] \mid d t \\
\leq \frac{1}{2(b-a)} L\left\|g^{\prime}\right\|_{\infty} \int_{a}^{b}(t-a)(b-t) d t \\
=\frac{1}{12} L(b-a)^{2}\left\|g^{\prime}\right\|_{\infty}
\end{aligned}
$$

where for the last inequality we used the inequality (15), with $d=t$ and $a=c$, (see (24)).

In the inequality (16), setting $d=t$ and $a=c$, we get

$$
\begin{aligned}
& \left|\frac{1}{t-a} \int_{a}^{t} g(s) d s-\frac{1}{b-a} \int_{a}^{b} g(s) d s\right| \\
& \quad \leq \begin{cases}\frac{(b-t)^{1+(1 / q)}}{(q+1)^{1 / q}(b-a)^{1 / q}} \\
\times\left[1+\left(\frac{t-a}{b+t-2 a}\right)^{q}\right]^{1 / q}\left\|g^{\prime}\right\|_{p} & g^{\prime} \in L_{p}[a, b], \\
& 1 \leq p<\infty, \\
\frac{1}{2} \frac{b-t}{b-a}\left\|g^{\prime}\right\|_{1}, & \frac{1}{p}+\frac{1}{q}=1 ; \\
& g^{\prime} \in L_{1}[a, b] .\end{cases}
\end{aligned}
$$

Substituting (37) into (36), we get

$|\mathscr{T}(f, g)|$

$$
\leq \frac{L}{b-a}\left\{\begin{array}{c}
\frac{\left\|g^{\prime}\right\|_{p}}{(q+1)^{1 / q}(b-a)^{1 / q}} \\
\times \int_{a}^{b}(t-a)(b-t)^{1+(1 / q)} \\
\quad \times\left[1+\left(\frac{t-a}{b+t-2 a}\right)^{q}\right]^{1 / q} d t \\
\frac{g^{\prime} \in L_{p}[a, b],}{2 \frac{\left\|g^{\prime}\right\|_{1}}{b-a} \int_{a}^{b}(t-a)(b-t) d t,} \\
g^{\prime} \in L_{1}[a, b],
\end{array}\right.
$$

$$
\begin{aligned}
& \leq \frac{L}{b-a}\left\{\begin{array}{c}
\frac{\left\|g^{\prime}\right\|_{p}}{(q+1)^{1 / q}(b-a)^{1 / q}} \\
\quad \times \sup _{t \in[a, b]}\left[1+\left(\frac{t-a}{b+t-2 a}\right)^{q}\right]^{1 / q} \\
\times \int_{a}^{b}(t-a)(b-t)^{1+(1 / q)} d t, \\
g^{\prime} \in L_{p}[a, b], \\
\frac{1}{12}(b-a)^{2}\left\|g^{\prime}\right\|_{1}, \\
g^{\prime} \in L_{1}[a, b],
\end{array}\right. \\
& =L \begin{cases}\frac{\left\|g^{\prime}\right\|_{p}}{(q+1)^{1 / q}}\left[1+\frac{1}{2^{q}}\right]^{1 / q} & \\
\times(b-a)^{2} \frac{q^{2}}{(2 q+1)(3 q+1)}, & g^{\prime} \in L_{p}[a, b], \\
\frac{1}{12}(b-a)\left\|g^{\prime}\right\|_{1}, & g^{\prime} \in L_{1}[a, b],\end{cases}
\end{aligned}
$$

where $\sup _{t \in[a, b]}\left[1+((t-a) /(b+t-2 a))^{q}\right]^{1 / q}=\left(1+\left(1 / 2^{q}\right)\right)^{1 / q}$, and

$$
\begin{aligned}
\int_{a}^{b}(t-a)(b-t)^{1+(1 / q)} d t \\
\quad=(b-a)^{3+(1 / q)} \int_{0}^{1}(1-t) t^{1+(1 / q)} d t \\
\quad=(b-a)^{3+(1 / q)} \frac{q^{2}}{(2 q+1)(3 q+1)},
\end{aligned}
$$

which proves the second and the third inequalities in (34).

Theorem 11. Let $f, g:[a, b] \rightarrow \mathbb{R}$ be such that $f$ is $L$ Lipschitzian on $[a, b]$ and $g$ is of $r$-H-Hölder type on $[a, b]$; then

$$
|\mathscr{T}(f, g)| \leq L H \frac{(b-a)^{r+1}}{(r+1)^{2}(r+2)} .
$$

Proof. As $f$ is $L$-Lipschitzian and $g$ is of $r$-H-Hölder type on $[a, b]$, by (36) and using (17) we have

$$
\begin{aligned}
& |\mathscr{T}(f, g)| \\
& \leq \frac{L}{b-a} \int_{a}^{b} \mid(t-a)\left[\frac{1}{t-a} \int_{a}^{t} g(u) d u\right. \\
& \left.\quad-\frac{1}{b-a} \int_{a}^{b} g(u) d u\right] \mid d t \\
& \leq \frac{L H}{(r+1)(b-a)} \int_{a}^{b}(t-a)(b-t)^{r} d t \\
& \quad=L H \frac{(b-a)^{r+1}}{(r+1)^{2}(r+2)}
\end{aligned}
$$


where, for the last inequality, a simple calculation yields that

$$
\begin{aligned}
\int_{a}^{b}(t-a)(b-t)^{r} d t & =(b-a)^{r+2} \int_{0}^{1}(1-t) t^{r} d t \\
& =\frac{(b-a)^{r+2}}{(r+1)(r+2)}
\end{aligned}
$$

which completes the proof.

Corollary 12. In Theorem 11, if $g$ is M-Lipschitzian on $[a, b]$, then we have

$$
|\mathscr{T}(f, g)| \leq \frac{1}{12} \operatorname{LM}(b-a)^{2}
$$

Theorem 13. Let $f, g:[a, b] \rightarrow \mathbb{R}$ be such that $f$ is of bounded variation on $[a, b]$ and $g$ is monotonic nondecreasing on $[a, b]$; then

$$
|\mathscr{T}(f, g)| \leq \frac{1}{6} L(b-a)^{2}[g(b)-g(a)] .
$$

Proof. As $f$ is $L$-Lipschitzian on $[a, b]$ and $g$ is monotonic nondecreasing on $[a, b]$, by (35) and using (36) we have

$$
\begin{aligned}
|\mathscr{T}(f, g)| \leq \frac{L}{b-a} \int_{a}^{b} \mid(t-a) & {\left[\frac{1}{t-a} \int_{a}^{t} g(u) d u\right.} \\
& \left.-\frac{1}{b-a} \int_{a}^{b} g(u) d u\right] \mid d t .
\end{aligned}
$$

In the third part of inequality (18), setting $d=t$ and $c=a$, we get

$\left|\frac{1}{t-a} \int_{a}^{t} g(s) d s-\frac{1}{b-a} \int_{a}^{b} g(s) d s\right| \leq \frac{b-t}{b-a}[g(b)-g(a)]$.

Substituting (46) into (45), we get

$$
\begin{aligned}
|\mathscr{T}(f, g)| & \leq \frac{L}{b-a} \frac{g(b)-g(a)}{b-a} \int_{a}^{b}(t-a)(b-t) d t \\
& =\frac{1}{6} L(b-a)[g(b)-g(a)],
\end{aligned}
$$

which completes the proof.

\section{More Inequalities}

In this section we give other related results.

Theorem 14. Let $f, g:[a, b] \rightarrow \mathbb{R}$ be such that $f$ and $g$ are of bounded variation on $[a, b]$; then

$$
|\mathscr{T}(f, g)| \leq \frac{1}{4} \bigvee_{a}^{b}(g) \bigvee_{a}^{b}(f) .
$$

The constant $1 / 4$ is the best possible.
Proof. As $f$ and $g$ is of bounded variation on $[a, b]$, by (22) and using (23), we have

$$
\begin{aligned}
& |\mathscr{T}(f, g)| \\
& \leq \frac{1}{b-a} \sup _{t \in[a, b]} \mid(t-a)\left[\frac{1}{t-a} \int_{a}^{t} g(u) d u\right. \\
& \left.-\frac{1}{b-a} \int_{a}^{b} g(u) d u\right] \mid \bigvee_{a}^{b}(f) \text {. }
\end{aligned}
$$

In the first inequality of (18), setting $d=t$ and $c=a$, we get

$$
\left|\frac{1}{t-a} \int_{a}^{t} g(s) d s-\frac{1}{b-a} \int_{a}^{b} g(s) d s\right| \leq \frac{b-t}{b-a} \bigvee_{a}^{b}(g) \text {. }
$$

Substituting (50) into (49), we get

$$
\begin{aligned}
|\mathscr{T}(f, g)| & \leq \frac{1}{(b-a)^{2}} \sup _{t \in[a, b]}\{(t-a)(b-t)\} \bigvee_{a}^{b}(g) \bigvee_{a}^{b}(f) \\
& =\frac{1}{4} \bigvee_{a}^{b}(g) \bigvee_{a}^{b}(f)
\end{aligned}
$$

which proves the inequality. The sharpness case trivially holds by taking $f(t)=g(t)=\operatorname{sgn}(t-((a+b) / 2))$, which completes the proof.

When the integrator is of bounded variation we have the following.

Theorem 15. Let $f, g:[a, b] \rightarrow \mathbb{R}$ be such that $f$ is $L$ Lipschitzian on $[a, b]$ and $g$ is of bounded variation on $[a, b]$; then

$$
|\mathscr{T}(f, g)| \leq \frac{1}{6} L(b-a) \bigvee_{a}^{b}(g) .
$$

Proof. As $f$ is $L$-Lipschitzian on $[a, b]$ and $g$ is of bounded variation on $[a, b]$, by (22) and using (23), we have

$$
\begin{aligned}
|\mathscr{T}(f, g)| \leq \frac{L}{b-a} \int_{a}^{b} \mid(t-a) & {\left[\frac{1}{t-a} \int_{a}^{t} g(u) d u\right.} \\
& \left.\quad-\frac{1}{b-a} \int_{a}^{b} g(u) d u\right] \mid d t .
\end{aligned}
$$

In the second inequality of (18), setting $d=t$ and $c=a$, we get

$$
\left|\frac{1}{t-a} \int_{a}^{t} g(s) d s-\frac{1}{b-a} \int_{a}^{b} g(s) d s\right| \leq \frac{b-t}{b-a} \bigvee_{a}^{b}(g) .
$$

Substituting (54) into (53), we get

$$
\begin{aligned}
|\mathscr{T}(f, g)| & \leq \frac{L}{(b-a)^{2}} \int_{a}^{b}(t-a)(b-t) d t \bigvee_{a}^{b}(g) \\
& =\frac{1}{6} L(b-a) \bigvee_{a}^{b}(g),
\end{aligned}
$$

and the proof is completed. 

ing.

When both functions are Lipschitzian we have the follow-

Theorem 16. Let $f, g:[a, b] \rightarrow \mathbb{R}$ be, respectively, such that $f$ and $g$ are $L_{1}$ - and $L_{2}$-Lipschitzian on $[a, b]$; then

$$
|\mathscr{T}(f, g)| \leq \frac{1}{12} L_{1} L_{2}(b-a)^{2} .
$$

The constant $1 / 12$ is the best possible.

Proof. As $f$ and $g$ are $L_{1}$ - and $L_{2}$-Lipschitzian on $[a, b]$, respectively, by (35) and using (36), we have

$$
\begin{aligned}
|\mathscr{T}(f, g)| \leq \frac{L_{1}}{b-a} \int_{a}^{b} \mid(t-a) & {\left[\frac{1}{t-a} \int_{a}^{t} g(u) d u\right.} \\
& \left.\quad-\frac{1}{b-a} \int_{a}^{b} g(u) d u\right] \mid d t .
\end{aligned}
$$

In the second inequality of (18), setting $d=t$ and $c=a$, we get

$$
\left|\frac{1}{t-a} \int_{a}^{t} g(s) d s-\frac{1}{b-a} \int_{a}^{b} g(s) d s\right| \leq \frac{1}{2} L_{2}(b-t) .
$$

Substituting (58) into (57), we get

$$
\begin{aligned}
|\mathscr{T}(f, g)| & \leq \frac{1}{2(b-a)} L_{1} L_{2} \int_{a}^{b}(t-a)(b-t) d t \\
& =\frac{1}{12} L_{1} L_{2}(b-a)^{2},
\end{aligned}
$$

which proves the inequality. The sharpness case trivially holds by taking $f(t)=g(t)=t$, which completes the proof.

Remark 17. Let $g$ be as in Theorems 7-16. By applying the same techniques used in the corresponding proofs of each theorem, we may obtain several inequalities for monotonic nondecreasing integrator $f$ using the fact that for a monotonic nondecreasing function $v:[a, b] \rightarrow \mathbb{R}$ and continuous function $p:[a, b] \rightarrow \mathbb{R}$, one has the inequality

$$
\left|\int_{a}^{b} p(t) d \nu(t)\right| \leq \int_{a}^{b}|p(t)| d \nu(t) .
$$

We leave the details to the interested reader.

\section{Applications for Self-Adjoint Operators}

We denote by $\mathscr{B}(H)$ the Banach algebra of all bounded linear operators on a complex Hilbert space $(H ;\langle\cdot, \cdot\rangle)$. Let $A \in$ $\mathscr{B}(H)$ be self-adjoint and let $\varphi_{\lambda}$ be defined for all $\lambda \in \mathbb{R}$ as follows:

$$
\varphi_{\lambda}(s):= \begin{cases}1, & \text { for }-\infty<s \leq \lambda \\ 0, & \text { for } \lambda<s<+\infty\end{cases}
$$

Then for every $\lambda \in \mathbb{R}$ the operator

$$
E_{\lambda}:=\varphi_{\lambda}(A)
$$

is a projection which reduces $A$.

The properties of these projections are collected in the following fundamental result concerning the spectral representation of bounded self-adjoint operators in Hilbert spaces; see for instance [24, page 256].

Theorem 18 (Spectral Representation Theorem). Let $A$ be a bonded self-adjoint operator on the Hilbert space $H$ and let $m=\min \{\lambda \mid \lambda \in \operatorname{Sp}(A)\}=: \min \operatorname{Sp}(A)$ and $M=\max \{\lambda \mid$ $\lambda \in \operatorname{Sp}(A)\}=: \max \operatorname{Sp}(A)$. Then there exists a family of projections $\left\{E_{\lambda}\right\}_{\lambda \in \mathbb{R}}$, called the spectral family of $A$, with the following properties:

(a) $E_{\lambda} \leq E_{\lambda^{\prime}}$ for $\lambda \leq \lambda^{\prime}$;

(b) $E_{m-0}=0, E_{M}=I$ and $E_{\lambda+0}=E_{\lambda}$ for all $\lambda \in \mathbb{R}$;

(c) we have the representation

$$
A=\int_{m-0}^{M} \lambda d E_{\lambda}
$$

More generally, for every continuous complex-valued function $\varphi$ defined on $\mathbb{R}$ and for every $\varepsilon>0$ there exists $a \delta>0$ such that

$$
\left\|\varphi(A)-\sum_{k=1}^{n} \varphi\left(\lambda_{k}^{\prime}\right)\left(E_{\lambda_{k}}-E_{\lambda_{k-1}}\right)\right\| \leq \varepsilon
$$

whenever

$$
\begin{gathered}
\lambda_{0}<m=\lambda_{1}<\cdots<\lambda_{n-1}<\lambda_{n}=M, \\
\lambda_{k}-\lambda_{k-1} \leq \delta \quad \text { for } 1 \leq k \leq n, \\
\lambda_{k}^{\prime} \in\left[\lambda_{k-1}, \lambda_{k}\right] \quad \text { for } 1 \leq k \leq n
\end{gathered}
$$

this means that

$$
\varphi(A)=\int_{m-0}^{M} \varphi(\lambda) d E_{\lambda}
$$

where the integral is of Riemann-Stieltjes type.

Corollary 19. With the assumptions of Theorem 18 for $A, E_{\lambda}$, and $\varphi$ we have the representations

$$
\varphi(A) x=\int_{m-0}^{M} \varphi(\lambda) d E_{\lambda} x \quad \forall x \in H,
$$

$$
\langle\varphi(A) x, y\rangle=\int_{m-0}^{M} \varphi(\lambda) d\left\langle E_{\lambda} x, y\right\rangle \quad \forall x, y \in H .
$$

In particular,

$$
\langle\varphi(A) x, x\rangle=\int_{m-0}^{M} \varphi(\lambda) d\left\langle E_{\lambda} x, x\right\rangle \quad \forall x \in H .
$$

Moreover, we have the equality

$$
\|\varphi(A) x\|^{2}=\int_{m-0}^{M}|\varphi(\lambda)|^{2} d\left\|E_{\lambda} x\right\|^{2} \quad \forall x \in H .
$$


We recall the following result (see [25]) that provides an upper bound for the total variation of the function $\mathbb{R} \ni \lambda \mapsto$ $\left\langle E_{\lambda} x, y\right\rangle \in \mathbb{C}$ on an interval $[\alpha, \beta]$.

Theorem 20. Let $\left\{E_{\lambda}\right\}_{\lambda \in \mathbb{R}}$ be the spectral family of the bounded self-adjoint operator $A$ and let $m=\min \operatorname{Sp}(\mathrm{A})$ and $M=$ $\max S p(\mathrm{~A})$. Then for any $x, y \in H$ and $\alpha<\beta$ we have the inequality

$$
\left[\bigvee_{\alpha}^{\beta}\left(\left\langle E_{(\cdot)} x, y\right\rangle\right)\right]^{2} \leq\left\langle\left(E_{\beta}-E_{\alpha}\right) x, x\right\rangle\left\langle\left(E_{\beta}-E_{\alpha}\right) y, y\right\rangle,
$$

where $\bigvee_{\alpha}^{\beta}\left(\left\langle E_{(\cdot)} x, y\right\rangle\right)$ denotes the total variation of the function $\left\langle E_{(\cdot)} x, y\right\rangle$ on $[\alpha, \beta]$.

Remark 21. For $\alpha=m-\varepsilon$ with $\varepsilon>0$ and $\beta=M$ we get from (70) the inequality

$$
\begin{aligned}
& \bigvee_{m-\varepsilon}^{M}\left(\left\langle E_{(\cdot)} x, y\right\rangle\right) \\
& \quad \leq\left\langle\left(1_{H}-E_{m-\varepsilon}\right) x, x\right\rangle^{1 / 2}\left\langle\left(1_{H}-E_{m-\varepsilon}\right) y, y\right\rangle^{1 / 2}
\end{aligned}
$$

for any $x, y \in H$.

This implies, for any $x, y \in H$, that

$$
\bigvee_{m-0}^{M}\left(\left\langle E_{(\cdot)} x, y\right\rangle\right) \leq\|x\|\|y\|
$$

where $\bigvee_{m-0}^{M}\left(\left\langle E_{(\cdot)} x, y\right\rangle\right)$ denotes the limit $\lim _{\varepsilon \rightarrow 0+}\left[\bigvee_{m-\varepsilon}^{M}\right.$ $\left.\left(\left\langle E_{(\cdot)} x, y\right\rangle\right)\right]$.

The inequality (72) was also proved in the recent monographs $[26,27]$ and will be utilized in the following.

After these preparations we can state and prove the following trapezoidal type inequality for functions of selfadjoint operators on Hilbert spaces.

Theorem 22. Let $A$ be a bonded self-adjoint operator on the Hilbert space $H$ and let $m=\min \{\lambda \mid \lambda \in \operatorname{Sp}(A)\}=$ : $\min S p(\mathrm{~A})$ and $M=\max \{\lambda \mid \lambda \in S p(A)\}=: \max S p(\mathrm{~A})$. If $f:[m, M] \rightarrow \mathbb{C}$ is such that its derivative $f$ is of bounded variation on $[m, M]$, then we have the inequality

$$
\begin{aligned}
& \left|\left\langle\left[f(A)-\frac{f(m)\left(M 1_{H}-A\right)+f(M)\left(A-m 1_{H}\right)}{M-m}\right] x, y\right\rangle\right| \\
& \quad \leq \frac{1}{4}(M-m) \bigvee_{m}^{M}(f) \bigvee_{m-0}^{M}\left(\left\langle E_{(\cdot)} x, y\right\rangle\right) \\
& \quad \leq \frac{1}{4}(M-m) \bigvee_{m}^{M}(f)\|x\|\|y\|
\end{aligned}
$$

for any $x, y \in H$.
Proof. Utilising the inequality (48) for the function of bounded variation $g(\lambda)=\left\langle E_{\lambda} x, y\right\rangle$ and the continuous function $f$ we have

$$
\begin{aligned}
& \mid \int_{m-0}^{M} f(\lambda) d\left\langle E_{\lambda} x, y\right\rangle-f(m) \frac{\int_{m-0}^{M}\left\langle E_{\lambda} x, y\right\rangle d \lambda}{M-m} \\
& -f(M)\left(\langle x, y\rangle-\frac{\int_{m-0}^{M}\left\langle E_{\lambda} x, y\right\rangle d \lambda}{M-m}\right) \mid \\
& \quad \leq \frac{1}{4}(M-m) \bigvee_{m}^{M}(f) \bigvee_{m-0}^{M}\left(\left\langle E_{(\cdot)} x, y\right\rangle\right) \\
& \quad \leq \frac{1}{4}(M-m) \bigvee_{m}^{M}(f)\|x\|\|y\|
\end{aligned}
$$

for any $x, y \in H$.

By the Spectral Representation Theorem we have

$$
\begin{aligned}
& \int_{m-0}^{M} f(\lambda) d\left\langle E_{\lambda} x, y\right\rangle=\langle f(A) x, y\rangle \\
& \int_{m-0}^{M}\left\langle E_{\lambda} x, y\right\rangle d \lambda=\left.\left\langle E_{\lambda} x, y\right\rangle \lambda\right|_{m-0} ^{M}-\int_{m-0}^{M} \lambda d\left\langle E_{\lambda} x, y\right\rangle \\
&=M\langle x, y\rangle-\langle A x, y\rangle \\
&=\left\langle\left(M 1_{H}-A\right) x, y\right\rangle
\end{aligned}
$$

for any $x, y \in H$.

Substituting these values into (74) we deduce after simple calculations the desired result (73).

The above inequality (73) can be utilized for different particular functions of interest in Operator Theory, such as the power, logarithmic, and exponential functions.

If we take $f(t)=t^{p}$ with $p \geq 1$, then for any positive operator $A$ with $\operatorname{Sp}(A) \subset[m, M] \subset[0, \infty)$ we have the inequality

$$
\begin{gathered}
\left|\left\langle\left[A^{p}-\frac{m^{p}\left(M 1_{H}-A\right)+M^{p}\left(A-m 1_{H}\right)}{M-m}\right] x, y\right\rangle\right| \\
\quad \leq \frac{1}{4}(M-m)\left(M^{p}-m^{p}\right) \bigvee_{m-0}^{M}\left(\left\langle E_{(\cdot)} x, y\right\rangle\right) \\
\quad \leq \frac{1}{4}(M-m)\left(M^{p}-m^{p}\right)\|x\|\|y\|
\end{gathered}
$$


If we take the function $f(t)=\ln t$, then for any positive definite operator $A$ with $\operatorname{Sp}(A) \subset[m, M] \subset(0, \infty)$ we have the inequality

$$
\begin{aligned}
& \left|\left\langle\left[\ln A-\frac{\ln m\left(M 1_{H}-A\right)+\ln M\left(A-m 1_{H}\right)}{M-m}\right] x, y\right\rangle\right| \\
& \quad \leq \ln \left(\frac{M}{m}\right)^{(M-m) / 4} \bigvee_{m-0}^{M}\left(\left\langle E_{(\cdot)} x, y\right\rangle\right) \\
& \quad \leq \ln \left(\frac{M}{m}\right)^{(M-m) / 4}\|x\|\|y\|
\end{aligned}
$$

for any $x, y \in H$.

Finally, if we take $f(t)=\exp (t)$, then we have for any selfadjoint operator $A$ with $\operatorname{Sp}(A) \subset[m, M] \subset \mathbb{R}$ the inequality

$$
\begin{aligned}
& \mid\langle[\exp (A) \\
& \left.\left.\quad-\frac{\exp (m)\left(M 1_{H}-A\right)+\exp (M)\left(A-m 1_{H}\right)}{M-m}\right] x, y\right\rangle \mid \\
& \quad \leq \frac{1}{4}(M-m)(\exp M-\exp m) \bigvee_{m-0}^{M}\left(\left\langle E_{(\cdot)} x, y\right\rangle\right) \\
& \quad \leq \frac{1}{4}(M-m)(\exp M-\exp m)\|x\|\|y\|
\end{aligned}
$$

for any $x, y \in H$.

\section{Conflict of Interests}

The author declares that there is no conflict of interests regarding the publication of this paper.

\section{References}

[1] P. L. Čebyšev, "Sur les expressions approximatives des intègrals dèfinis par les outres prises entre les même limites," Proceedings of the Mathematical Society of Kharkov, vol. 2, pp. 93-98, 1882.

[2] G. Grüss, "Über das Maximum des absoluten Betrages von $1 /(b-a) \int_{a}^{b} f(x) g(x) d x-1 /(b-a)^{2} \int_{a}^{b} f(x) d x \int_{a}^{b} g(x) d x$, Mathematische Zeitschrift, vol. 39, no. 1, pp. 215-226, 1935.

[3] D. S. Mitrinović, J. E. Pečarić, and A. M. Fink, Classical and New Inequalities in Analysis, Kluwer Academic Publishers, Dordrecht, The Netherlands, 1993.

[4] A. M. Ostrowski, "On an integral inequality," Aequationes Mathematicae, vol. 4, no. 3, pp. 358-373, 1970.

[5] A. Lupaş, “The best constant in an integral inequality," Mathematica, vol. 15, no. 38, pp. 219-222, 1973.

[6] S. S. Dragomir, "New Grüss' type inequalities for functions of bounded variation and applications," Applied Mathematics Letters, vol. 25, no. 10, pp. 1475-1479, 2012.

[7] M. W. Alomari, "Some Grüss type inequalities for RiemannStieltjes integral and applications," Acta Mathematica Universitatis Comenianae, vol. 81, no. 2, pp. 211-220, 2012.
[8] M. W. Alomari and S. S. Dragomir, "Mercer-Trapezoid rule for Riemann-Stieltjes integral with applications," Journal of Advances in Mathematics, vol. 2, no. 2, pp. 67-85, 2013.

[9] M. W. Alomari, "A companion of Ostrowski's inequality for the Riemann-Stieltjes integral $\int_{a}^{b} f(t) d u(t)$, where $f$ is of bounded variation and $u$ is of $r-H$-Hölder type and applications," Applied Mathematics and Computation, vol. 219, no. 9, pp. 4792-4799, 2013.

[10] M. W. Alomari, "Difference between twoStieltjes integral means," Kragujevac Journal of Mathematics. In press.

[11] M. W. Alomari, "A sharp bound for the Čebyšev functional of convex or concave functions," Chinese Journal of Mathematics, vol. 2013, Article ID 295146, 3 pages, 2013.

[12] M. W. Alomari, "New sharp inequalities of Ostrowski and generalized trapezoid type for the Riemann-Stieltjes integrals and applications," Ukrainian Mathematical Journal, vol. 65, no. 7, pp. 995-1018, 2013.

[13] M. W. Alomari, "New Grüss type inequalities for double integrals," Applied Mathematics and Computation, vol. 228, pp. 102-107, 2014.

[14] M. W. Alomari and S. S. Dragomir, "New Grüss type inequalities for Riemann-Stieltjes integral with monotonic integrators and applications," Annals of Functional Analysis, vol. 5, no. 1, pp. 7793, 2014.

[15] M. W. Alomari and S. S. Dragomir, "Some Grüss type inequalities for the Riemann-Stieltjes integral with Lipschitzian integrators," Konuralp Journal of Mathematics. In press.

[16] S. S. Dragomir, "A generalisation of Cerone's identity and applications," Tamsui Oxford Journal of Mathematical Sciences, vol. 23, no. 1, pp. 79-90, 2007.

[17] S. S. Dragomir, "Inequalities for Stieltjes integrals with convex integrators and applications," Applied Mathematics Letters, vol. 20, no. 2, pp. 123-130, 2007.

[18] S. S. Dragomir, "Accurate approximations of the RiemannStieltjes integral with (1,L)-Lipschitzian integrators," AIP Conference Proceedings, vol. 936, no. 1, p. 686, 2007.

[19] S. S. Dragomir, "Sharp Grüss-type inequalities for functions whose derivatives are of bounded variation," Journal of Inequalities in Pure and Applied Mathematics, vol. 8, no. 4, article 117, 2007.

[20] S. S. Dragomir, "Bounds for some perturbed Čebyšev functionals," Journal of Inequalities in Pure and Applied Mathematics, vol. 9 , no. 3, article 64, 2008.

[21] B. G. Pachpatte, "On Čebyšev type inequalities involving functions whose derivatives belong to $L_{p}$ spaces," Journal of Inequalities in Pure and Applied Mathematics, vol. 7, no. 2, article 58,2006

[22] N. S. Barnett, P. Cerone, S. S. Dragomir, and A. M. Fink, "Comparing two integral means for absolutely continuous mappings whose derivatives are in $L_{\infty}[a, b]$ and applications," Computers and Mathematics with Applications, vol. 44, no. 1-2, pp. 241-251, 2002.

[23] P. Cerone and S. S. Dragomir, "Differences between Means with Bounds from a Riemann-Stieltjes Integral," Computers and Mathematics with Applications, vol. 46, no. 2-3, pp. 445-453, 2003.

[24] G. Helmberg, Introduction to Spectral Theory in Hilbert Space, John Wiley \& Sons, New York, NY, USA, 1969.

[25] S. S. Dragomir, "Some trapezoidal vector inequalities for continuous functions of selfadjoint operators in Hilbert spaces," Acta Mathematica Vietnamica, 2014. 
[26] S. S. Dragomir, Operator Inequalities of the Jensen, Čebyšev and Grüss Type, Springer Briefs in Mathematics, Springer, New York, NY, USA, 2012.

[27] S. S. Dragomir, Operator Inequalities of Ostrowski and Trapezoidal Type, Springer Briefs in Mathematics, Springer, New York, NY, USA, 2012. 


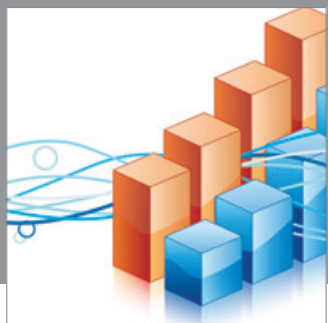

Advances in

Operations Research

mansans

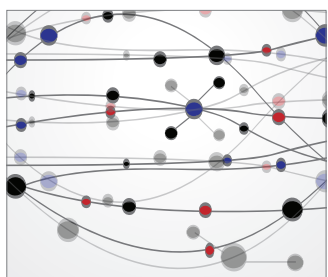

The Scientific World Journal
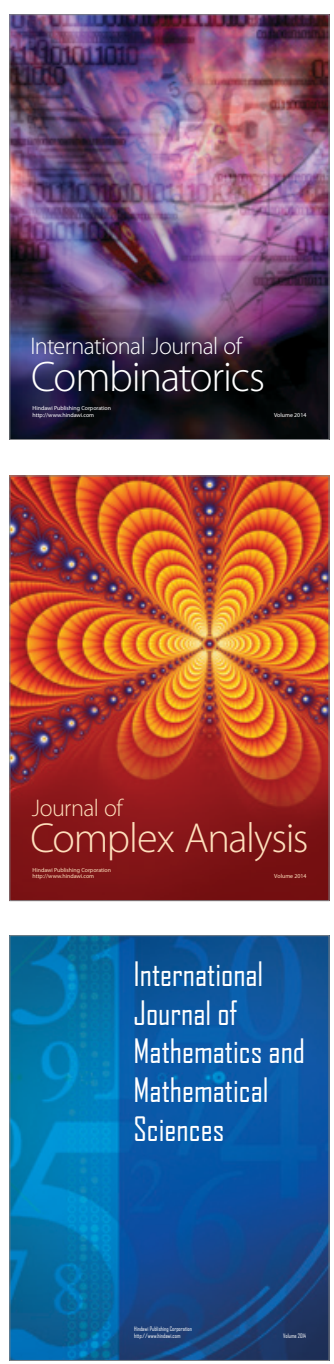
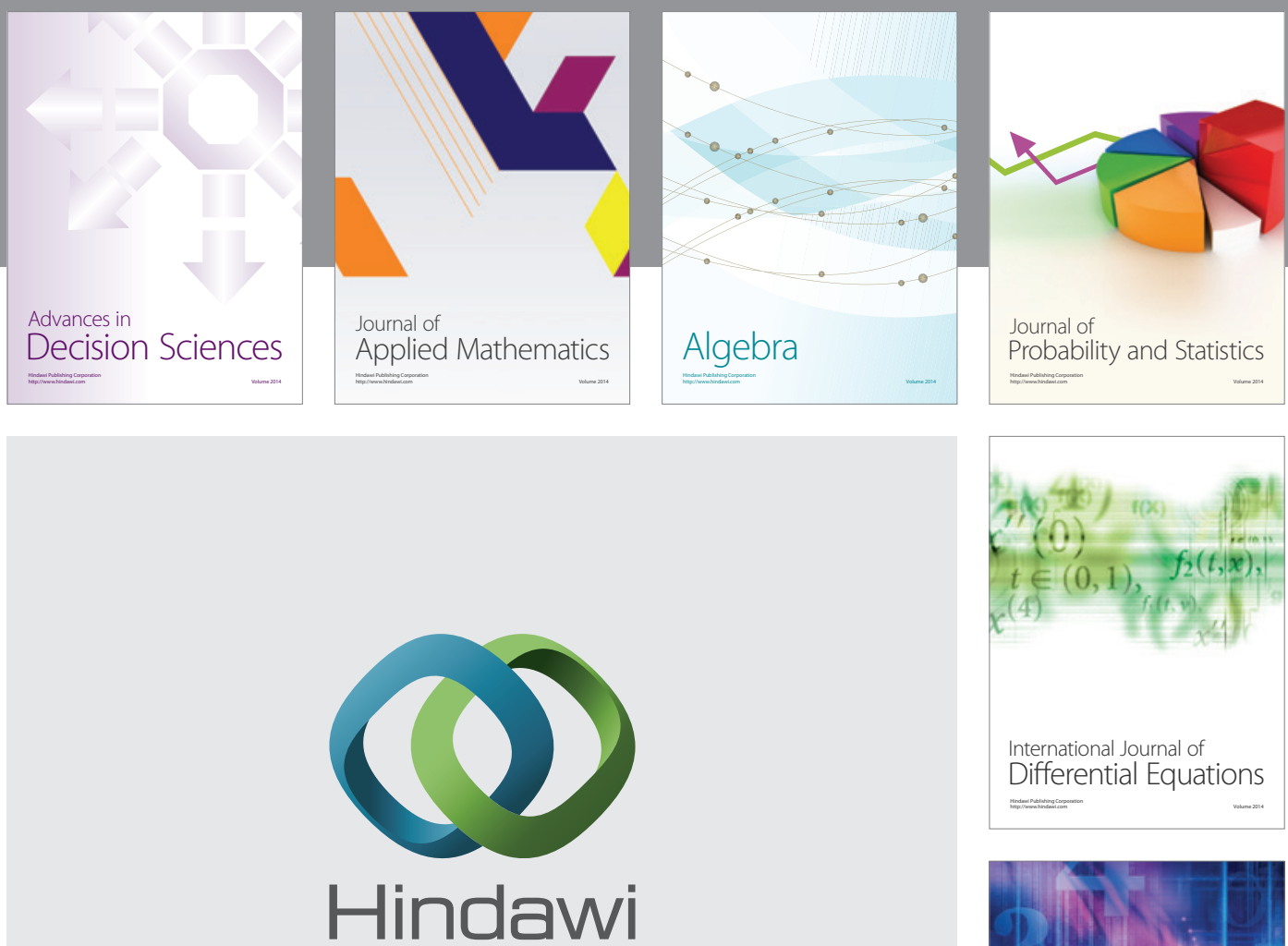

Submit your manuscripts at http://www.hindawi.com


Journal of

Function Spaces

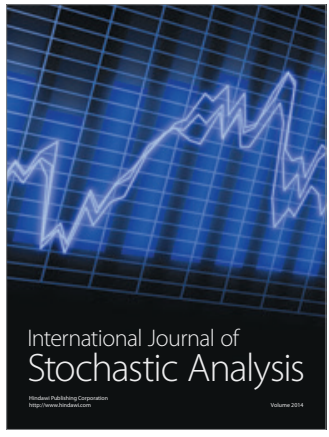

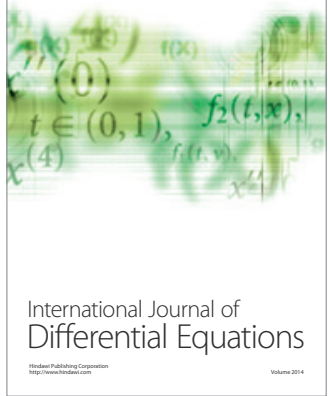
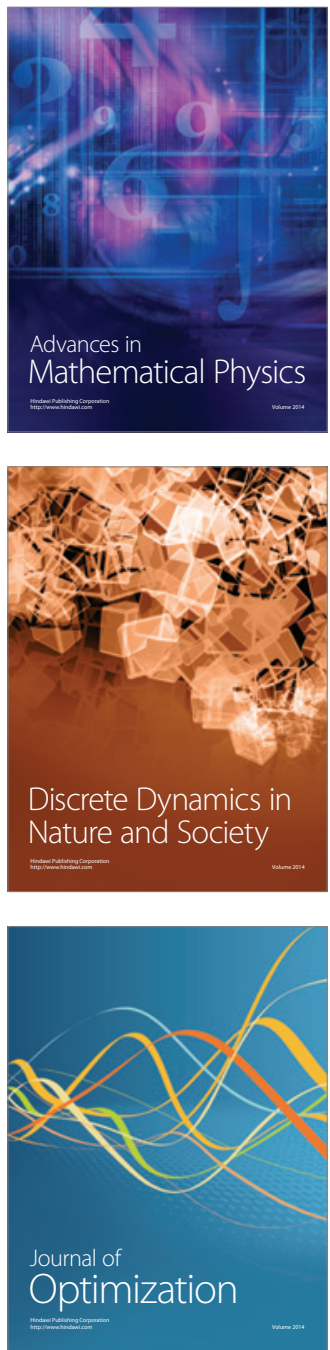